\title{
Polarization Coupling of Light and Optoelectronics Devices Based on Periodically Poled Lithium Niobate
}

\author{
Xianfeng Chen*, Kun Liu, and Jianhong Shi \\ Shanghai Jiao Tong University,
}

China

\section{Introduction}

Ferroelectric crystals are important materials in many kinds of applications. Since the invention of so called quasi-phase matching technique, periodically domain-inverted ferroelectrics crystals with modulated second order nonlinear susceptibility has received much attention owing to its outstanding nonlinear optical properties(Magel et al.,1990; Xu et al.,1993; Zheng et al.,1998). In periodically domain-inverted ferroelectrics crystals, besides the nonlinear optical coefficients, other third-rank tensors, such as the electro-optic (EO) coefficient, are also modulated periodically because of the periodically reversed ferroelectric domains. Since the theoretical research on EO effect in PPLN was proposed by Y.Q Lu et al(Lu et al.,2000), this periodically modulation of the EO coefficient has given birth to some novel essential applications, especially when the voltage is applied along the transverse direction of the crystal. In this chapter, current research of transverse EO-based PPLN applications, such as tunable wavelength filter, polarization controller, electro-optical switch and laser Q-switch in periodically poled lithium niobate are summarized.

\section{Solc-type wavelength filter}

Tunable optical filters play an important role in optical signal processing and optical communication. In particular, a narrow flat-top bandpass filter, which not only enables a particular wavelength channel to pass through but also significant to keep signal stable, as well as a multiple-wavelength filter, which enables several wavelengths to pass through together at the same time, are widely needed in wavelength-division multiplexing systems. In this section, we present our research on tunable filter with single-wavelength, multiplewavelength and flat-top passband based on PPLN crystal. Photovoltaic effect in PPLN is also investigated and based on which a tunable filter by use of UV light is demonstrated. Moreover, the latest researches on solc-type filter based on Ti:PPLN channel waveguide are also presented in the end of this section.

\subsection{Principles}

When an electric field is applied along the $Y$ axis of a crystal with $3 \mathrm{~m}$ symmetry, the new index ellipsoid deforms to make its principal axes rotated at an angle $\theta$ about the $x$ axis with 
respect to the unperturbed principal axes. Since $\theta$ is proportional to the product of the electric field and the electro-optic coefficient, when an uniformed electric field applying along the $\mathrm{Y}$ axis of the crystal, the rotation angle of the original domain is opposite to that of the inverted domain because of the reversal of the spontaneous polarization( $\mathrm{Lu}$ et al.,2000). Thus, in a periodically domain inverted crystal with a uniformed electric field applied along the $\mathrm{Y}$ axis, a structure with alternating left and right rotation angle $\theta$ will be formed due to the periodic EO coefficient. This structure is similar to the well known folded Solc filter structure with alternating azimuth angles of the crystal axes. So we call it Solc layered structure.

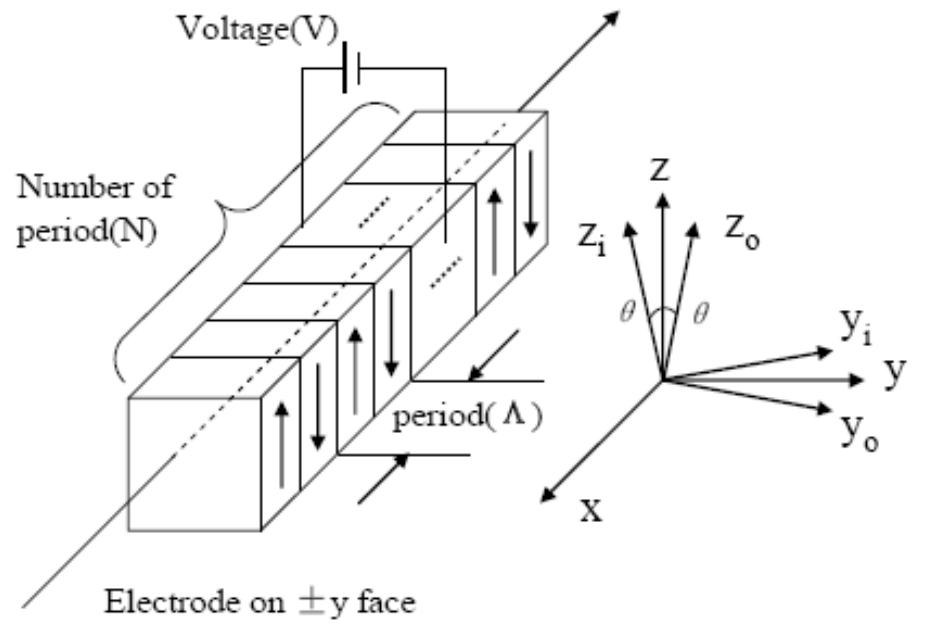

Fig. 1. Schematic diagram of a Solc layered structure based on electro-optic periodically domain inverted crystals. $X, Y$ and $Z$ are the principal axes of the unperturbed index ellipsoid. $\Lambda$ is the period of this structure and $N$ is the period number. The arrows inside the structure indicate the spontaneous polarization directions. $Y_{o}, Z_{o}$ and $Y_{i}, Z_{i}$ are the new perturbed principal axes of the original domains and the inverted domains with external electric field, respectively(Lu et al.,2000).

To have an insight into the filter process, polarization coupled-mode theory is established to track the polarization state of light propagation along PPLN. In PPLN the coupled-wave equations of the ordinary and extraordinary waves are:

$$
\left\{\begin{array}{l}
\mathrm{dA}_{1} / \mathrm{dx}=-i \kappa \mathrm{A}_{2} \exp (\mathrm{i} \Delta \beta \mathrm{x}) \\
\mathrm{dA}_{2} / \mathrm{dx}=-i \kappa^{*} \mathrm{~A}_{1} \exp (-\mathrm{i} \Delta \beta \mathrm{x})
\end{array}\right.
$$

with $\Delta \beta=\left(k_{2}-k_{1}\right)-G_{m}, G_{m}=2 \pi m / \Lambda$ and $\kappa=-\frac{\omega}{2 c} \frac{n_{o}^{2} n_{e}^{2} \gamma_{51} E_{y}}{\sqrt{n_{\mathrm{o}} n_{e}}} \frac{i(1-\cos m \pi)}{m \pi} \quad(m=1,3,5 \ldots)$, where $A_{1}$ and $\mathrm{A}_{2}$ are the normalized amplitudes of the ordinary wave and the extraordinary wave, respectively, $k_{1}$ and $k_{2}$ are the corresponding wave vectors, $G_{m}$ is the $m$ th reciprocal vector corresponding to the periodicity of poling. Consider an input light with Y-axis polarization direction, the boundary condition of the equation is given by 


$$
\left\{\begin{array}{l}
\mathrm{A}_{1}(0)=1 \\
\mathrm{~A}_{2}(0)=0
\end{array}\right.
$$

So the solution of coupled-mode equation (1) is:

$$
\left\{\begin{array}{l}
A_{1}(x)=\exp [i(\Delta \beta / 2) x][\operatorname{cossx}-i(\Delta \beta / 2 s) \sin s x] \\
A_{2}(x)=\exp [-i(\Delta \beta / 2) x]\left(-i \kappa^{*}\right) \operatorname{sinsx} / s
\end{array}\right.
$$

where $\mathrm{s}$ is given by $\mathrm{s}^{2}=\kappa \kappa^{*}+(\Delta \beta / 2)^{2}$.

When $\Delta \beta=\left(\mathrm{k}_{2}-\mathrm{k}_{1}\right)-\mathrm{G}_{\mathrm{m}}=0$, the reciprocal vector compensates for the wave-vector mismatch. We call this condition a QPM polarization conversion condition, which determines the fundamental wavelength of this kind filter. Under QPM condition, the transmission along the Z-axis is given by

$$
\mathrm{T}=\left|\mathrm{A}_{2}(\mathrm{x}) / \mathrm{A}_{1}(0)\right|^{2}=\sin ^{2}(\mathrm{Sx})
$$

If $|\mathrm{Sx}|=|\kappa| \mathrm{L}=(2 \mathrm{~m}+1) \pi / 2,(\mathrm{~m}=0,1,2 \ldots)$, then $\mathrm{T}=100 \%$ and the power of the input light is completely transferred from $\mathrm{Y}$ polarization to $\mathrm{Z}$ polarization. Thereby, with a polarizer along Z-axis at the output, the fundamental wavelength will pass it without any loss, while at the same time wavelengths which don't satisfy this condition will be forbidden by the analyzer. The working mechanism of solc-type filter can also be easily understood as follows: Because each domain serves as a half-wave plate with respect to the fundamental wavelength. After passing through the stack of half-wave plates, the optical plane of polarization of the fundamental wavelength rotates continually and emerges finally at an angle of $2 \mathrm{~N} \theta$, where $\mathrm{N}$ is the number of plates. Therefore, when $2 \mathrm{~N} \theta=90^{\circ}$ at the filter output, light of the fundamental wavelength does not experience loss in passing through the crossed analyzer.

\subsection{Device of tunable Solc-type wavelength filter \\ 2.2.1 Tunable single-wavelength filter}

The first observation of a Solc filter based on PPLN operating in the optical communication wavelength range was proposed by our group in 2003 (Chen et al., 2003). Similar to the traditional folded type Solc filter, a PPLN crystal is placed between two crossed polarizer. This sample is with dimensions of $28 \mathrm{~mm} \times 5 \mathrm{~mm} \times 0.5 \mathrm{~mm}$ consists of four gratings with periods from 20.2 to $20.8 \mu \mathrm{m}$ and a width of $1 \mathrm{~mm}$. A measured transmission power versus wavelength for $20.6 \mu \mathrm{m}$ and $20.8 \mu \mathrm{m}$ period is shown in Fig.3, from which a typical transmission spectrum of traditional Solc-type filter can be seen. The amplitude modulation of the transmission power by applying an electric voltage along the $Y$ axis of the PPLN is also observed and the result is shown in Fig.4. In above discussion, we have known that the fundamental wavelength is determined by equation $\Delta \beta=\left(\mathrm{k}_{2}-\mathrm{k}_{1}\right)-\mathrm{G}_{\mathrm{m}}=0$, which can be simplified to $\lambda_{0}=\Lambda\left(\mathrm{n}_{\mathrm{o}}-\mathrm{n}_{\mathrm{e}}\right)$. Because the refractive indices of the ordinary wave and the extraordinary wave are temperature dependent, yet the fundamental wavelength can be shifted to a series of different wavelengths by changing the temperature. The experiment 


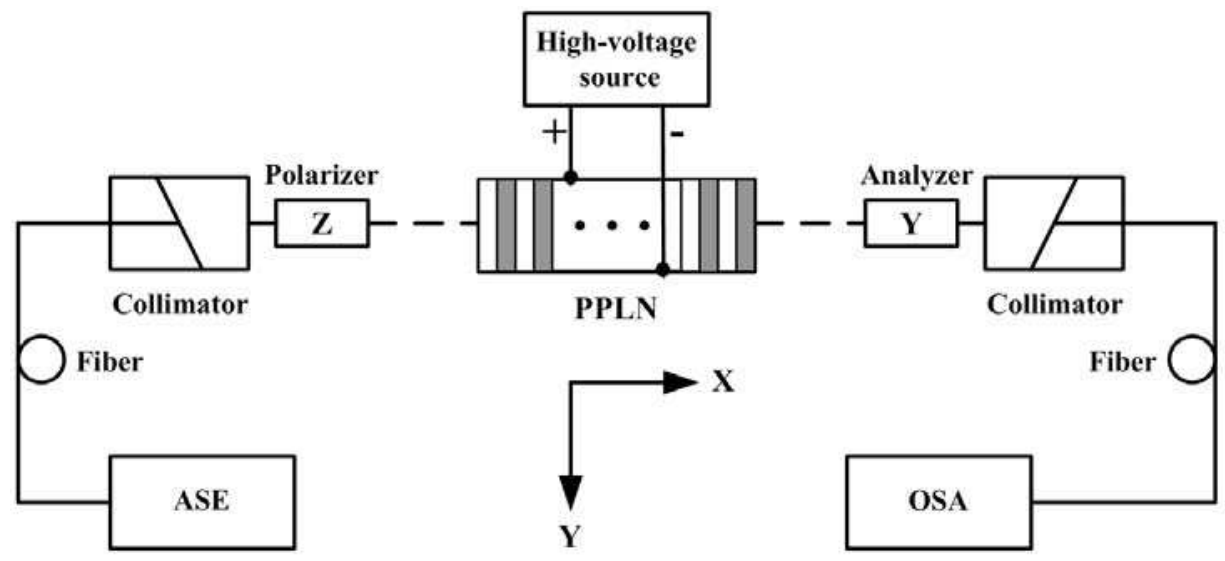

Fig. 2. Experimental setup for a PPLN single-wavelength filter. A PPLN crystal, which is Z cut, is placed between two crossed polarizer, the first of which is along $\mathrm{Z}$ direction and the second $Y$ direction. The light propagates along the $X$ direction and a uniform electric field is applied along the $\mathrm{Y}$ axis of the PPLN sample. ASE, amplified spontaneous emission; OSA, optical spectrum analyzer(Chen et al.,2003).

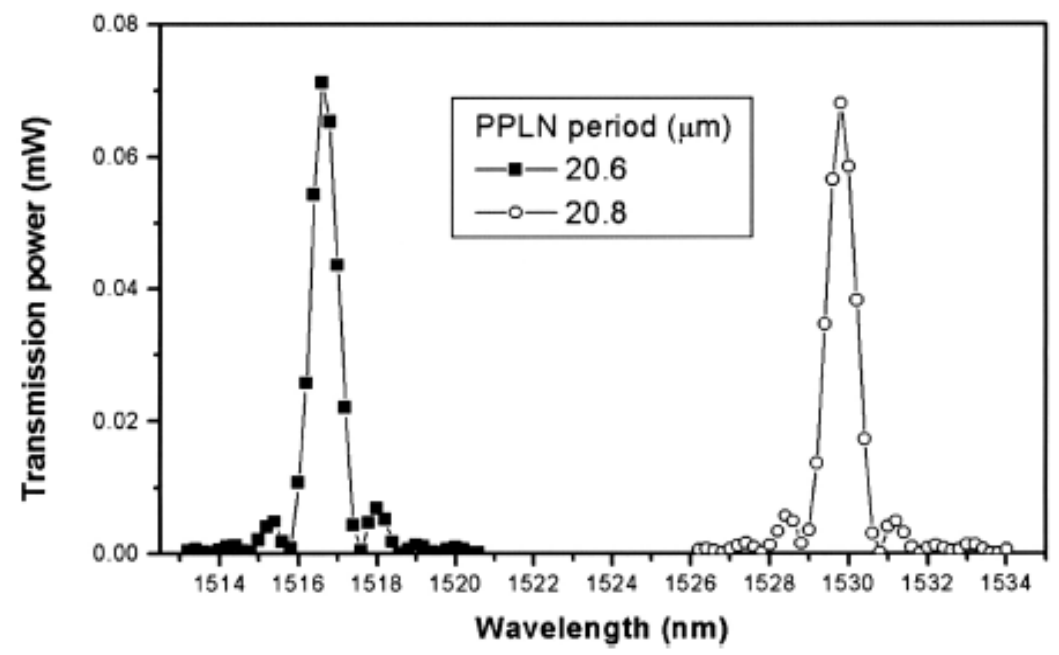

Fig. 3. Measured transmission power versus wavelength of the Solc filter in the 20.6- and 20.8-mm-period PPLN at a temperature of $24^{\circ} \mathrm{C}$. The FWHM of the transmission spectrum is $0.8 \mathrm{~nm}$ (Chen et al.,2003).

result on wavelength modulation is showed in Fig.5, where $-0.415 \mathrm{~nm} /{ }^{\circ} \mathrm{C}$ tuning rate is achieved. Wavelength tuning by temperature is interesting in dense wavelength division multiplexer optical fiber communication systems, where it can be employed as a wavelength-tunable filter for all-optical wavelength routing. 


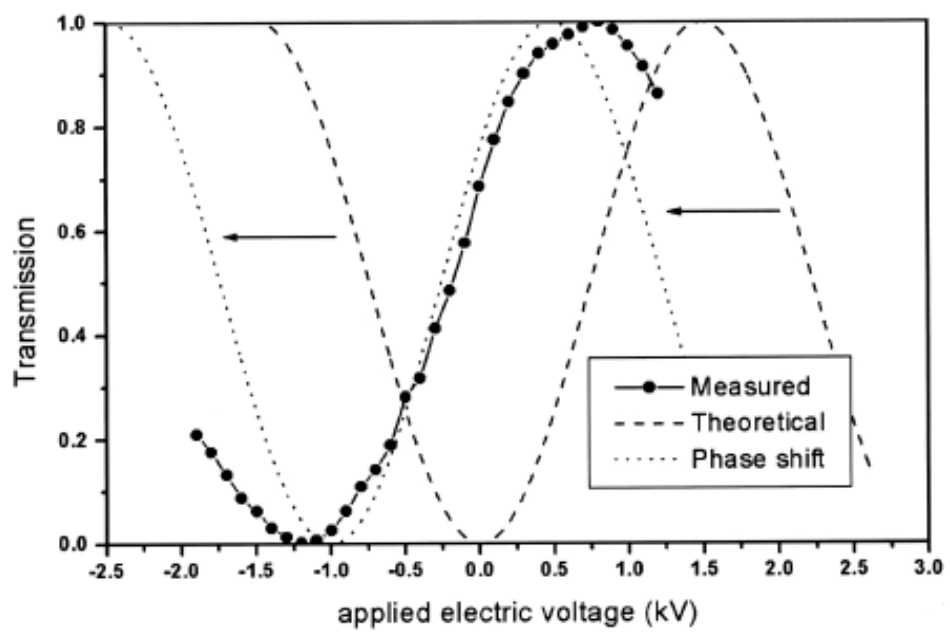

Fig. 4. Experimental measurement of the normalized transmission of the Solc filter (20.8 $\mathrm{mm}$ ) as a function of the applied voltage at $24{ }^{\circ} \mathrm{C}$ for a given wavelength of $1529.80 \mathrm{~nm}$. The solid curve is the experimental measurement, the dashed curve represents the theoretical values calculated from the Sellmeier equation(Jundt,1997), and the dotted curve is phase shifted from the dashed curve for comparison with the experiment(Chen et al.,2003).

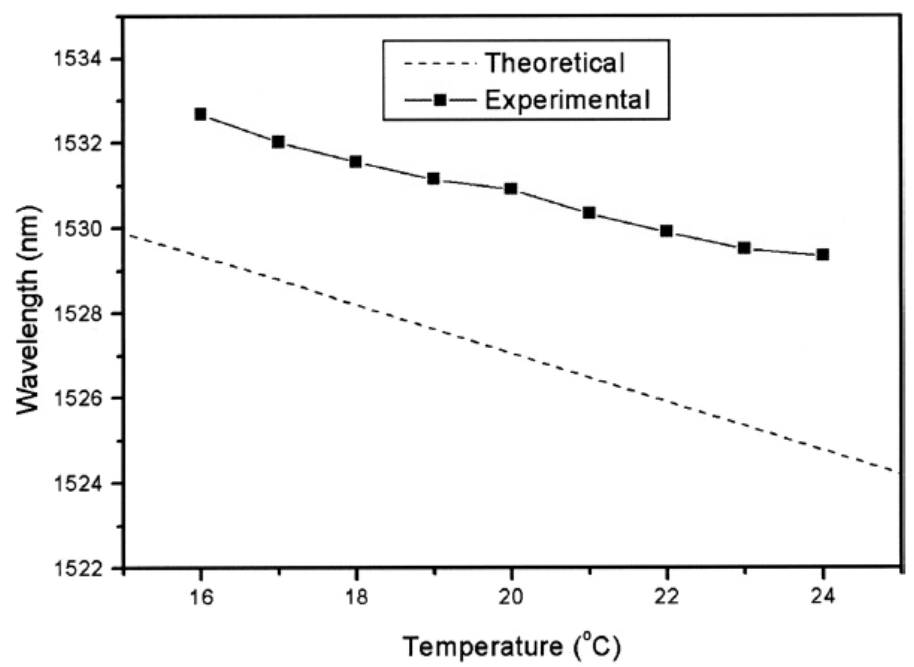

Fig. 5. Experimental measurement of the central wavelength of the Solc $\mathrm{f}$ ilter $(20.8 \mathrm{~mm})$ as a function of the temperature without the applied external electric voltage. The dashed line represents the theoretical values calculated from the Sellmeier equation(Jundt,1997), and the solid curve is the experimental measurement(Zhu et al.,2003). 


\subsubsection{Tunable multiple-wavelength filter}

In previous section, we have discussed that the central wavelength of a PPLN Solc-type filter is determined by the period of the domain inversion and the ordinary and extraordinary refractive indices. Since the indices are temperature dependent, the central wavelength of such filter can be shifted by changing the working temperature of the whole PPLN. If we apply a temperature distribution along the PPLN through a local-temperaturecontrol technique to control and reshape the second harmonic (SH) curve, then the PPLN will be divided into several temperature sections and each section will give a central wavelength determined by its local temperature. By combining the contributions from all the temperature sections in the PPLN, a multi-wavelength filter can be realized. By properly controlling the temperature of each section, we can construct a tunable multi-wavelength filter for any wavelength according to the practical needs of different applications(Wang et al.,2007).
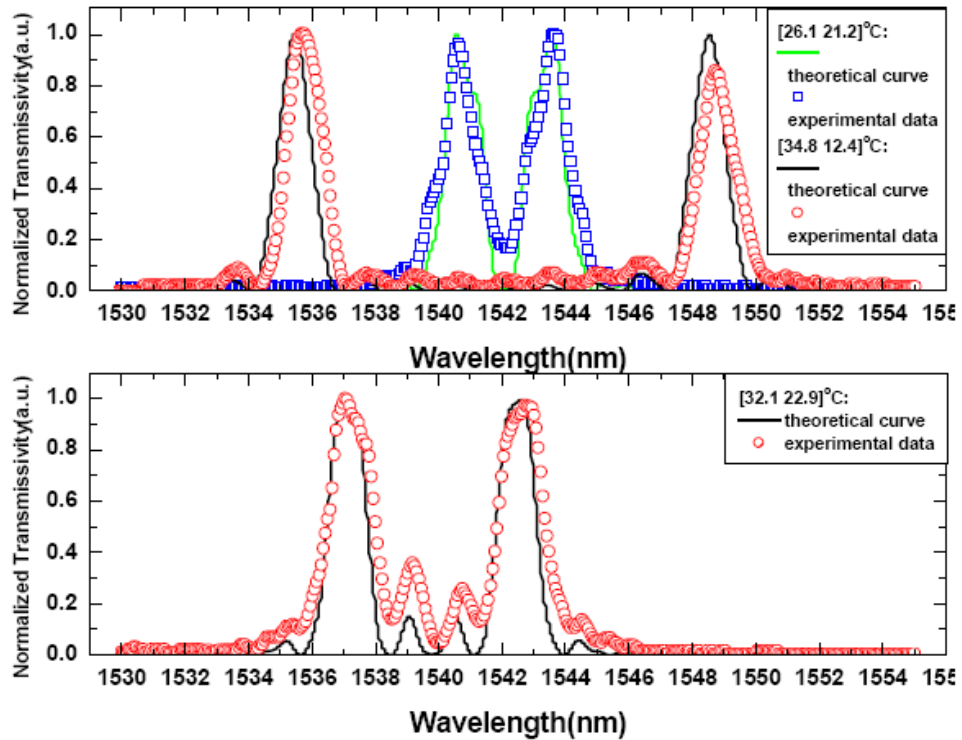

Fig. 6. Tunable double-wavelength filter realized in PPLN by applying a two-section pattern temperature distribution along the sample. In the first figure, under a [26.1 21.2] ${ }^{\circ} \mathrm{C}$ pattern temperature distribution, the double peaks were at $1540.616 \mathrm{~nm}$ and $1543.63 \mathrm{~nm}$, and under a [34.8 12.4] ${ }^{\circ} \mathrm{C}$ pattern temperature distribution, the double peaks were tuned to $1538.562 \mathrm{~nm}$ and $1545.6845 \mathrm{~nm}$, for the symmetrical case. In the second figure, under a [32.1 22.9] ${ }^{\circ} \mathrm{C}$ pattern temperature distribution, the double peaks were set to $1537.078 \mathrm{~nm}$ and $1542.763 \mathrm{~nm}$, for the arbitrary case(Wang et al.,2007).

The multiple-wavelength filter can also been designed by optimizing the sequences of the opposite domains in aperiodic poled lithium niobate (APLN) using simulated annealing (SA) algorithm(Gu et al.,2004). Here, Jones matrix, a $2 \times 2$-matrix method, is employed to track the polarization state of light propagation along APLN (Yariv \& Yeh,1984). The sequence of the positive and negative domains is optimized with the target that the 
transmission of the prescribed wavelengths are equal with maximum values at the same time. Here, we chose the objective function in the SA method as

$$
\mathrm{F}=\sum_{\alpha} \mathrm{T}\left(\lambda_{\alpha}\right)-\left\{\max \left[\mathrm{T}\left(\lambda_{\alpha}\right)\right]-\min \left[\mathrm{T}\left(\lambda_{\alpha}\right)\right]\right\}
$$

where the symbol $\max [\ldots](\min [\ldots])$ manifests to take their maximum (minimum) value along all the quantities including into [...]. $\lambda_{\alpha}$ is the transmission wavelength selected as the objective wavelength in the function. In our calculation, the length of each domain is set to be $5 \mu \mathrm{m}$. The length $\mathrm{L}$ of lithium niobate is $5 \mathrm{~cm}$ and the number of blocks is $N=10000$. The wavelength dependent refractive indices of extraordinary wave and ordinary wave are calculated at $20^{\circ} \mathrm{C}$ from the Sellmeier equation(Jundt,1997). Wavelengths 1548.5, 1550.1, 1551.7, and $1553.3 \mathrm{~nm}$ are chosen as the filtering wavelengths of APLN filter. These wavelengths are from ITU standard wavelength table for DWDM optical communication, with frequency spacing of $200 \mathrm{GHz}$. Fig.7 shows the filtering results of two, three and four 200-GHz-spaced DWDM wavelengths by simulated annealing algorithm.
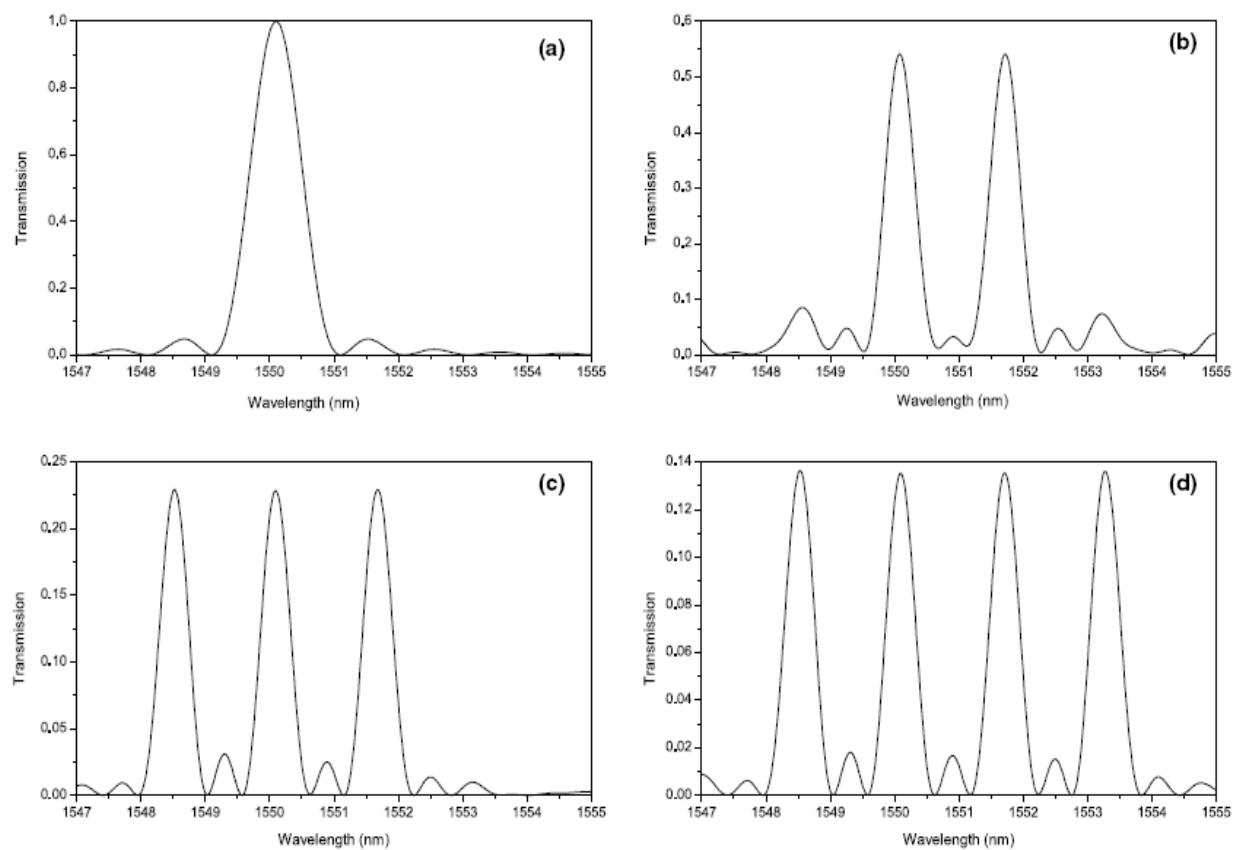

Fig. 7. Calculated transmission spectra of narrowband multiple wavelengths filters at $20^{\circ} \mathrm{C}$. The length L of lithium niobate is $5 \mathrm{~cm}$. (a) a spectrum of PPLN Solc filter. (b)- (d) transmission spectrums with two, three and four peaks in APLN filters optimized by SA method. The number of domains is $\mathrm{N}=10000$ and the applied electric field in $\mathrm{Y}$-direction is $70 \mathrm{~V} / \mathrm{mm}$. The peak wavelengths are 1548.5, 1550.1, 1551.7 and $1553.3 \mathrm{~nm}$, respectively $(\mathrm{Gu}$ et al.,2004).

The first experimental observation of narrowband multi-wavelength filters based on aperiodically poled lithium niobate crystals was reported by C. H. Lin et.al(C.H.Lin 
et.al.,2007). In the experiment, they simultaneously transmitted 8 ITU-standard wavelengths through a 5-cm long EO APLN filter with a transmittance of $>90 \%$ and a bandwidth of $\sim 0.45$ $\mathrm{nm}$ for each wavelength channel. Moreover, the transmission spectrum of the EO APLN filter can be conveniently tuned by temperature at a rate of $\sim 0.65 \mathrm{~nm} /{ }^{0} \mathrm{C}$ in the telecom $\mathrm{C}$ band.

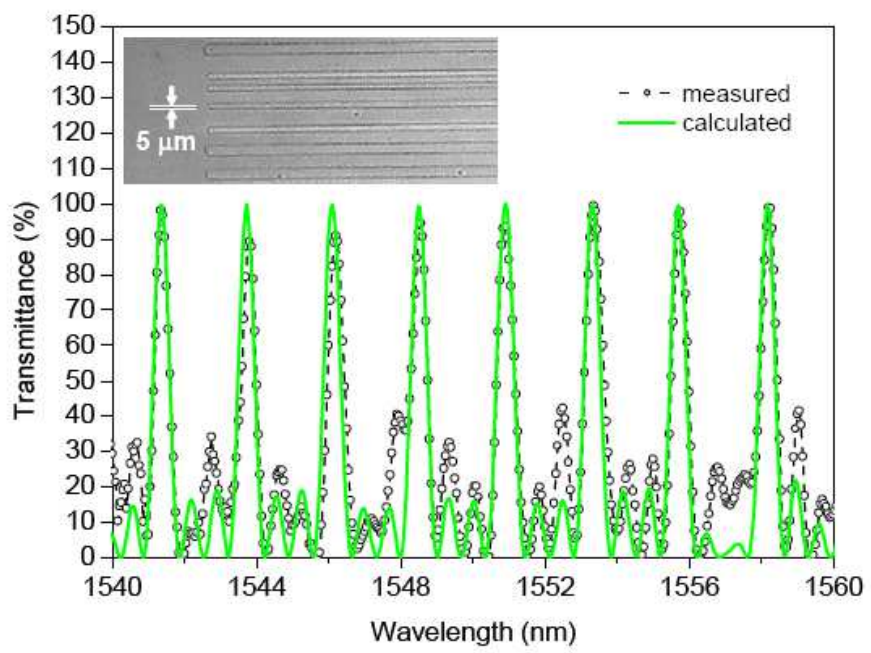

Fig. 8. Calculated (solid line) and measured (open circles) transmission spectra of the 5-cm long EO APLN filter. The two spectral curves agree reasonably well, except that the side lobes in the experimental curve are more apparent. The inset shows a microscopic image of an HF-etched z surface of the fabricated APLN crystal. The label in the inset indicates the width of the unit domain block used in this APLN crystal, i.e., $\Delta x=5 \mu \mathrm{m}$ (C.H.Lin et al.,2007).

\subsubsection{Flat-top wavelength filter}

Generally, the passband of this kind filter is extremely narrow. Tiny shift of the fundamental wavelength because of unstable temperature or any other factors will contribute to great loss. Thereby, a flat-top filter which can avoid the interference of the outside and maintain signal quality is necessary.

To begin with this section, we would like to introduce the spectrum waveform similarity (SWS) rule discovered in PPLN firstly(Liu et al.,2009a). The SWS rule can be described as follows: if the product of the domain $\mathrm{N}$ and the rocking angle $\theta$ is fixed, the shape of the spectrum waveform remains similar, as $\mathrm{N}$ varies. To get a clear picture of this rule, Fig. 9 will be helpful. In figure 9, " $\mathrm{A}$ " represents the product of the domain $\mathrm{N}$ and the rocking angle $\theta$. With " $\mathrm{A}$ " fixed at pi/2, the shape of the spectrum waveform remains similar when $\mathrm{N}$ is changing, despite the FWHM correspondingly increases. Based on the spectrum waveform similarity (SWS) rule, we come to a conclusion that the shape of the spectrum waveform is determined by the product $\mathrm{A}$. By observing the spectrums at different value of A, our study reveals a critical point $(A=2.24)$ at which the spectrum surprisingly evolved into flat-top type (Fig.10). The $1 \mathrm{~dB}$ width of the flat-top waveform can be fitted as follows: 


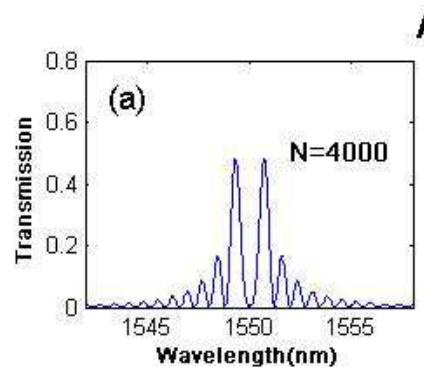

\section{$A=\pi / 2$}
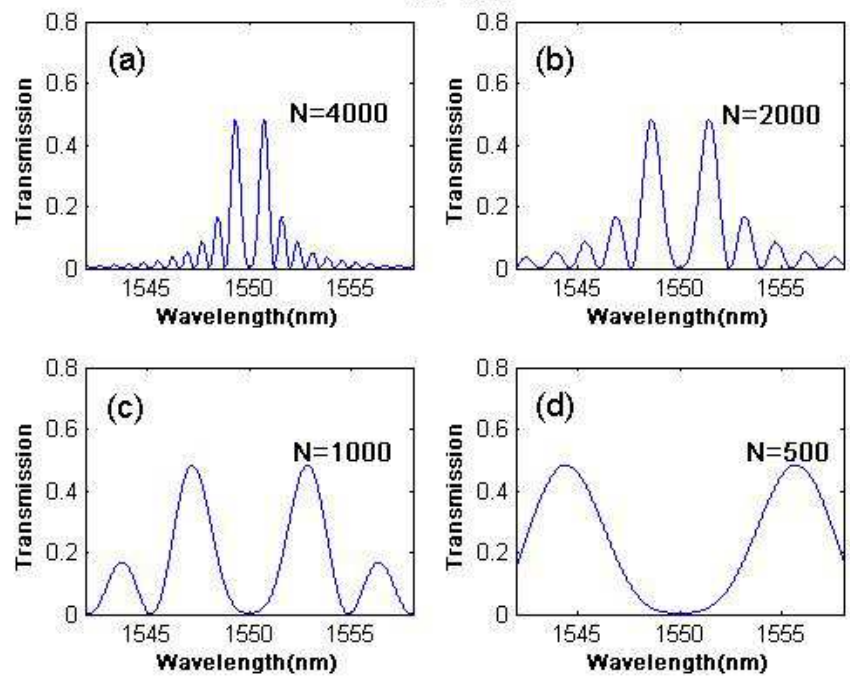

Fig. 9. The transmission spectrums by use of numeral calculation in PPLN with the product A fixed at $\pi / 2$. (a) The transmission spectrum with $\mathrm{N}$ at 4000; (b) The transmission spectrum with $\mathrm{N}$ at 2000; (c) The transmission spectrum with $\mathrm{N}$ at 1000; (d) The transmission spectrum with $\mathrm{N}$ at 500(Liu et al.,2009a).

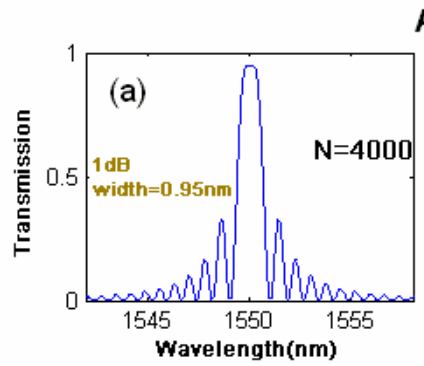

\section{$A=2.24$}
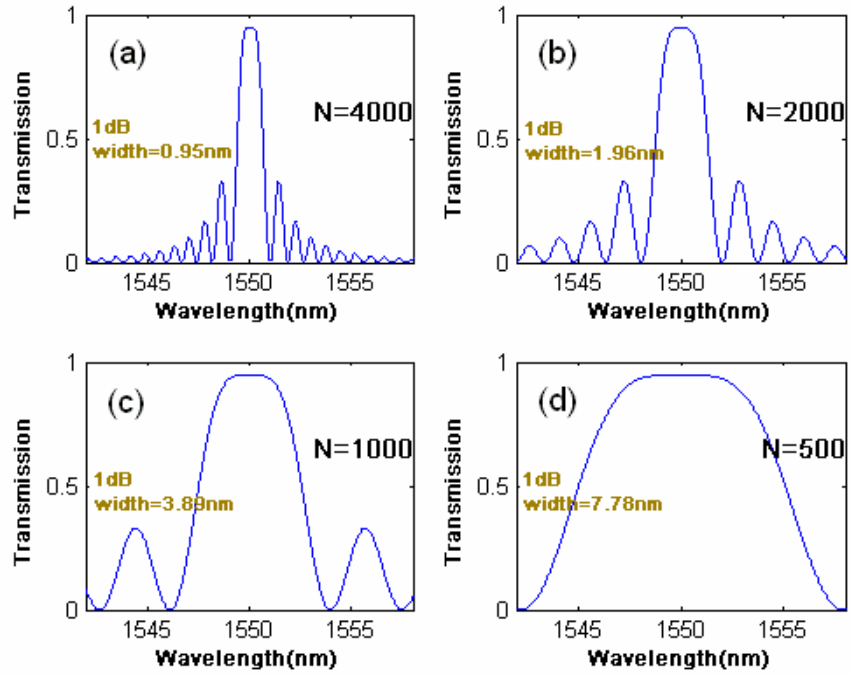

Fig. 10. The transmission spectrums by use of numeral calculation in PPLN with the product A fixed at 2.24. (a) The transmission spectrum with $\mathrm{N}$ at 4000; (b) The transmission spectrum with $\mathrm{N}$ at 2000; (c) The transmission spectrum with $\mathrm{N}$ at 1000; (d) The transmission spectrum with $\mathrm{N}$ at 500(Liu et al.,2009a). 


$$
\Delta \lambda_{\text {ldb }} \gg 2.5 \lambda_{0} / \mathrm{N}
$$

where $\lambda_{0}$ is the fundamental wavelength of the filter.

In order to confirm this theoretical discovery about flat-top spectrum in PPLN, further investigation has been performed experimentally(Liu et.al,2009b). The PPLN sample, employed in the experiment, with a dimension of $30(\mathrm{~L}) \times 10(\mathrm{~W}) \times 0.5(\mathrm{~T}) \mathrm{mm}^{3}$ consists of 2857 domains with the period of $21(\mu \mathrm{m})$. In above discussion, we have known the flat-top spectrum requires $\mathrm{N} \theta=2.24$ which is greater than the single wavelength Solc-type filter $(2 \mathrm{~N} \theta=л / 2)$. Thereby, as to a given PPLN ( $\mathrm{N}$ fixed), higher electric field is requested than usual. During the experiment, the transmission of the fundamental wavelength attends to maximum at $3(\mathrm{kV} / \mathrm{cm})(\mathrm{A})$ and gradually declines when the electric field keeps on arising subsequently. Consistent with the theoretical result, the spectrum evolves into flat-top type with higher electric field at the critical point $4.2(\mathrm{kV} / \mathrm{cm})(B)$. The corresponding transmission spectrums at A and B are, respectively, shown in Fig.11. A flat-top waveform with $1(\mathrm{~nm})$ flat-top width is obtained in the experiment.

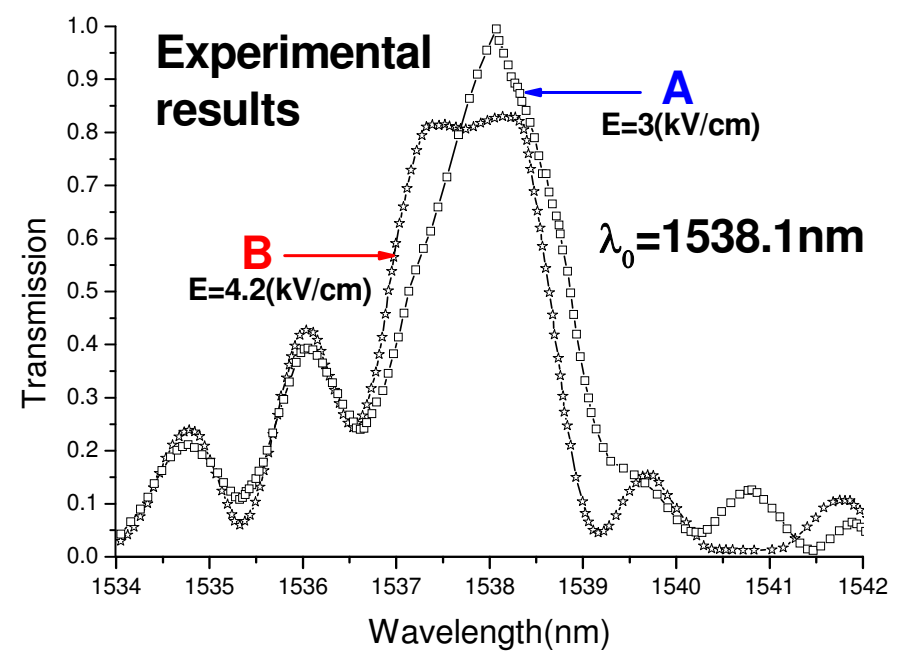

Fig. 11. The transmission spectrums at electric fields of A and B. The curve with square symbol represents the spectrum with $3(\mathrm{kV} / \mathrm{cm})$ electric field $(\mathrm{A})$ and the curve with star symbol represents the spectrum with $4.2(\mathrm{kV} / \mathrm{cm}$ ) electric field (B) (Liu et al.,2009b).

Interestingly, as to a given PPLN, by extending the external electric field to a higher value, the theoretical results reveal that new critical points of flat-top will be found every 2.4 $(\mathrm{kV} / \mathrm{cm})$ electric field. Besides, the width of each flat-top transmission spectrum is proportionally increased with the increment of external electric field. Thereby, tunable passband can be achieved by switching the external electric field between different critical points which is shown in Fig.12.

In this section, both the theory and the experiment show that there is a critical voltage at which a flat-top transmission spectrum can be achieved. To have an insight into the flat-top process, more studies should proceed in future. We believe this phenomenon may be also found in other materials and structures similar to PPLN. As the flat-top spectrum is able to 
keep signal stable, this finding may improve the possibility of the practical application of Solc-type PPLN filter in optical networks and optical signal processing in future.

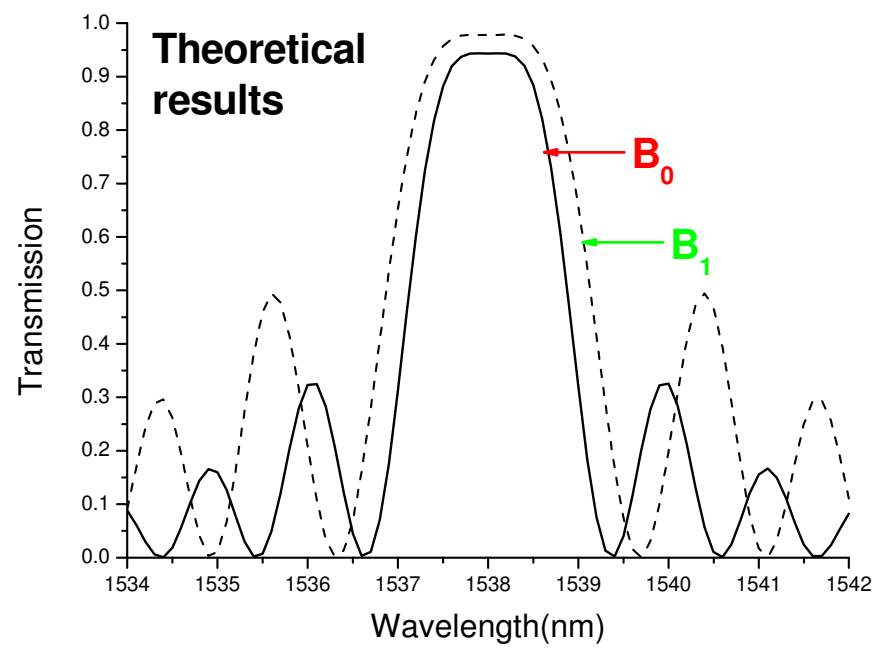

Fig. 12. The theoretical transmission spectrums at critical points $B_{0}$ and $B_{1}$. The solid curve represents the theoretical spectrum at $B_{0}$; The dash curve represents the theoretical spectrum at $B_{1}$ (Liu et al.,2009b).

\subsubsection{Photovoltaic effect in PPLN crystal}

In the experiments of above sections, we also found that, without field applied, the device also has the function of Solc filter, which shows disagreement with the theory. Our study reveals that the photovoltaic effect (PVE) plays an essential role in the performance of nonfield applied PPLN Solc filter(Chen et al.,2006). The PVE effect sometimes can not be neglected, especially in electric-optic devices. Our theoretical analysis shows that only the

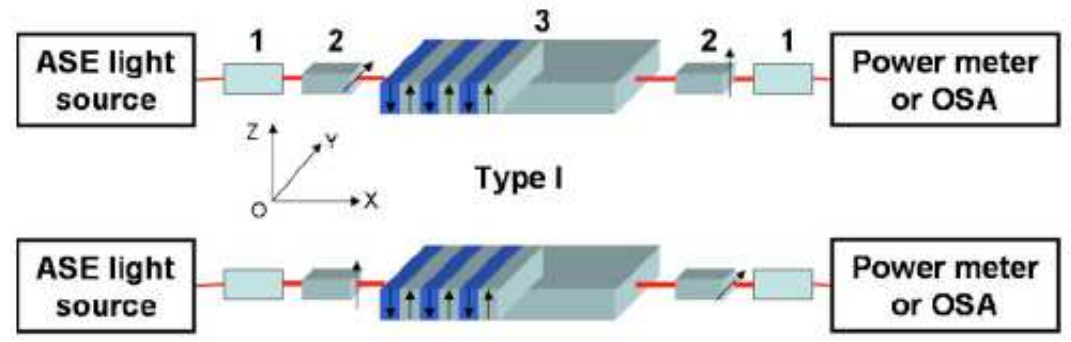

Type II

Fig. 13. Two types of device setups for PPLN Solc-type wavelength filter; the arrow on the polarizer indicates the polarization direction. The fact that type A takes on the filter behavior while type B does not reveals the test light has the PVE on the PPLN and it becomes a Solc filter when no external field exists. The numbered components are: 1 , collimator; 2, crossed polarizer; 3, PPLN(Chen et al.,2006). 
electric field $\mathrm{E}$ in the $\mathrm{Y}$ direction can enable the Solc filter. If the input polarizer is put in the $Y$ direction, there will be a field formed in the $Y$ direction that will make the device work as a Solc filter; Whereas, if the input polarizer is set in the $\mathrm{Z}$ direction, no field will cause the half-wave plate rocking and the PPLN will have no filter function. By observing the spectrums with two types of setups shown in Fig.13, the theoretical analysis is experimentally confirmed in Fig.14.

On the other hand, this PVE effect inspires us a new thought to build the light controlled optical devices or to measure the PVE coefficients (Shi et al., 2006). Fig.15 shows measured

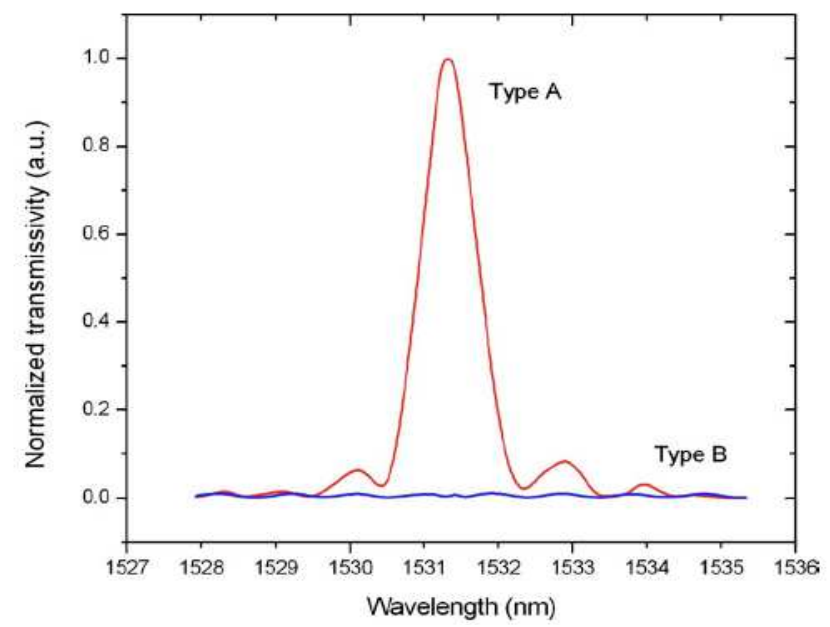

Fig. 14. The transmission curve of the type A setup of device is in accord with the Solc filter function while the type B setup almost has no pass peak(Chen et al.,2006).

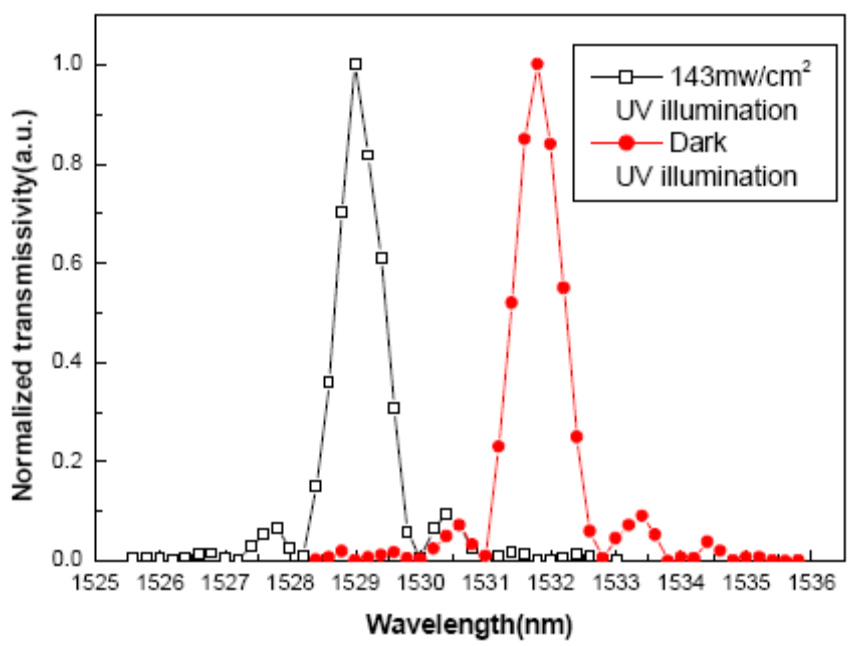

Fig. 15. The spectrum of the PPLN filter under $143 \mathrm{~mW} / \mathrm{cm}^{2} \mathrm{UV}$ illumination (empty square symbol) and without UV light illumination (red circle symbol) (Shi et al.,2006). 
output power against wavelength for the PPLN of period $20.8 \mu \mathrm{m}$ with and without the illumination of the UV light, respectively. The UV light intensity is about $143 \mathrm{mw} / \mathrm{cm}^{2}$. The passband of the filter moves from 1531.9nm to $1529.1 \mathrm{~nm} .4$ ). It costs several seconds until spectrum of the filter becomes stable. However, the peak value and the shape of the pass band are unchanged. It means the $Y$ field of the PVE is not changed. This suggests that no $Y$ direction field is induced when a non-polarized light propagates in $\mathrm{Z}$ direction.

\subsubsection{Solc-type filter in Ti:PPLN channel waveguide}

In this section, we will present the latest research on solc-type filter based on Ti:PPLN channel waveguide which has a significant advantage of low driven voltage. The filtering mechanism of this kind filter is same to the filter based on PPLN crystal introduced above. In this kind filter, the gap between the electrodes can be as short as $(\mu \mathrm{m})$ level, therefore, only several voltages are enough for filtering or switching a light(Lee et al.,2007). The experimental setup to perform the wavelength filtering based on the Ti:PPLN channel waveguide is shown in Fig.16. An optical signal from a wavelength-swept fiber laser based on a semiconductor optical amplifier and a Fabry-Perot tunable filter was collimated and end-fire coupled into the Ti:PPLN waveguide, which is placed between two crossed polarizer by a $10 x$ objective lens. The polarization direction of input beam was adjusted parallel to the $\mathrm{Y}$ axis of the Ti:PPLN device, and the output signal was observed by an optical spectrum analyzer. The filtering results of one-mode guiding condition and twomode guiding condition are shown in Fig. 17. In one-mode condition, the measured FWHM of the filter was about $0.21 \mathrm{~nm}$, and in two-mode condition, the origin of one peak is the TEM00-like mode, and the other is the TEM01-like mode. The wavelength difference of the two peaks is about $0.8 \mathrm{~nm}$.

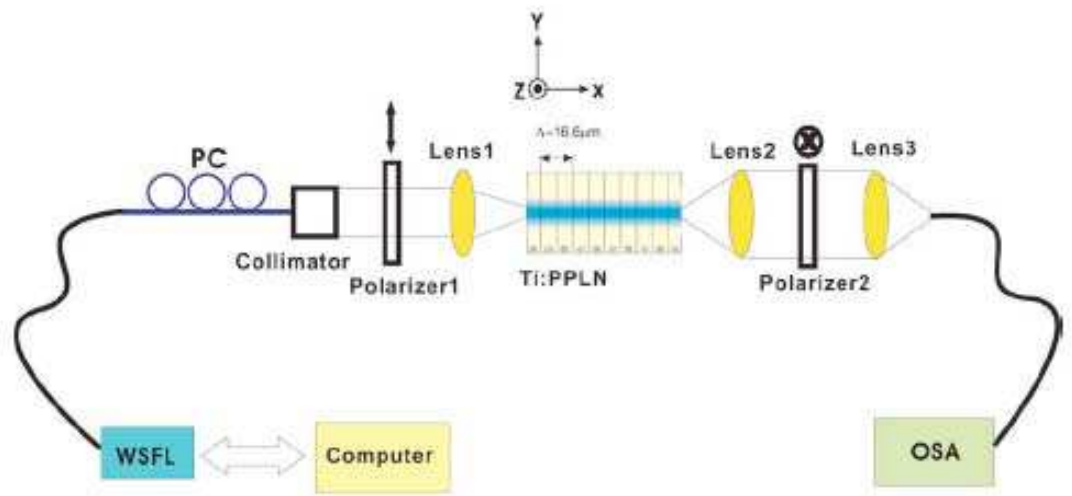

Fig. 16. (Color online) Experimental setup for the waveguide-type solc-type filter based on the Ti:PPLN channel waveguide. WSFL, wavelength-swept fiber laser; OSA, optical spectrum analyzer; PC, polarization controller(Lee et al.,2007).

Similar to the light controlled optical solc-type filter by use of PVE effect in PPLN crystal, Y.L. Lee et.al demonstrate a tunable all-optical solc-type wavelength filter based on Ti:PPLN channel waveguide(Lee et al.,2008). Fig.18 shows the centre wavelength of the filter as a function of the UV illumination intensity. As the intensity increases, the centre wavelength of the filter shifts to a shorter wavelength. The measured wavelength tuning rate of the filter 


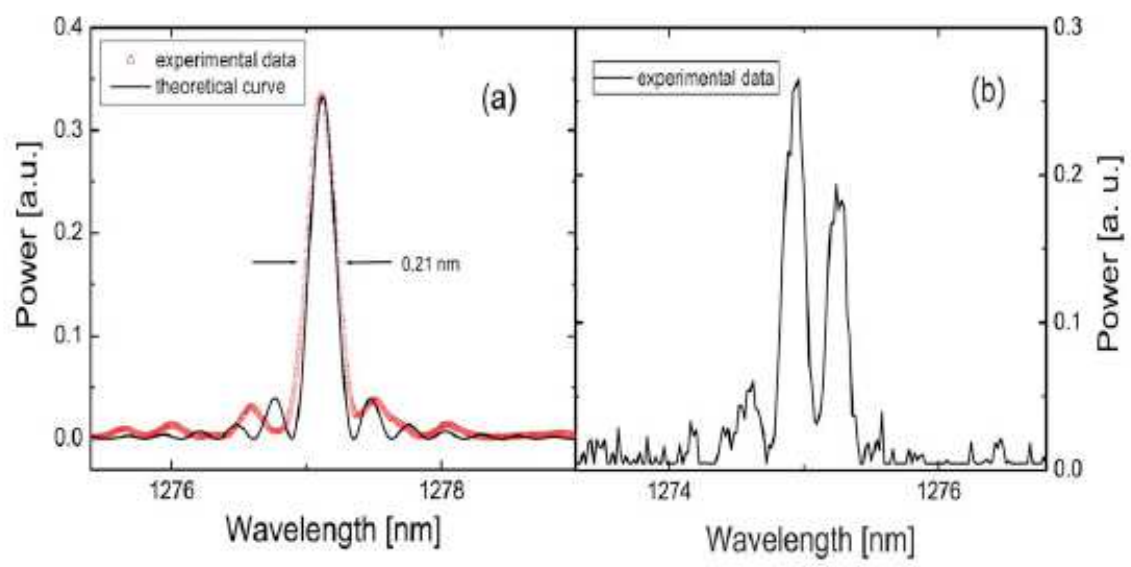

Fig. 17. (Color online) Optical spectra of the Šolc filter based on the Ti:PPLN waveguide depending on guiding modes at room temperature: (a) one-mode guiding condition, (b) two-mode guiding condition(Lee et al.,2007).

was about $-26.42 \mathrm{~nm} / \mathrm{W} \mathrm{cm}{ }^{-2}$ which shows more slant than that of a bulk PPLN Solc-type filter $\left(-19.23 \mathrm{~nm} / \mathrm{W} \mathrm{cm}^{-2}\right.$ ) (Shi et al.,2006). The right axis of Fig.18 indicates the amount of change in the refractive index difference between $n_{o}$ and $n_{e}$ as a function of UV intensity. These results indicate that a Ti:PPLN solc-type filter gives a wider wavelength tuning range than that of a bulk PPLN solc-type filter.

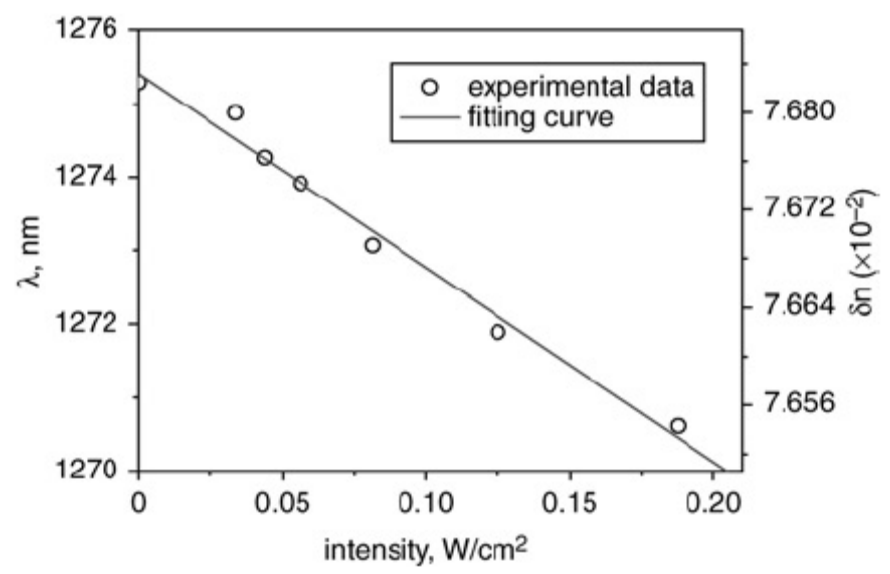

Fig. 18. Centre wavelength of Ti:PPLN solc-type filter aganst UV illumination intensity(Lee et al., 2008)

\section{Polarization controller}

In this section, we will introduce our study on precise linear polarization-state controller based on PPLN(Liu et al.,2009c), which was also inspired by Y.Q. Lu et.al.'s study(Lu et 
al.,2000), where they theoretically proposed the electro-optical effect in PPLN. The working mechanism is the same with the Solc-type filter. It is known that with respect to the fundamental wavelength of solc-type filter, each domain serves as a half-wave plate. Therefore, after passing through the stack of half-wave plates, the optical plane of polarization of the fundamental wavelength rotates continually and emerges finally at an angle of $2 \mathrm{~N} \theta$. As $\theta$ is controlled with external electric field, the final rotation angle is as well dominated by the electric field. Consequently, an EO linear polarization-state controller for the fundamental wavelength can be realized. In the following discussion, we use another name of "operating wavelength" to represent the fundamental wavelength which is more appropriate than the latter.

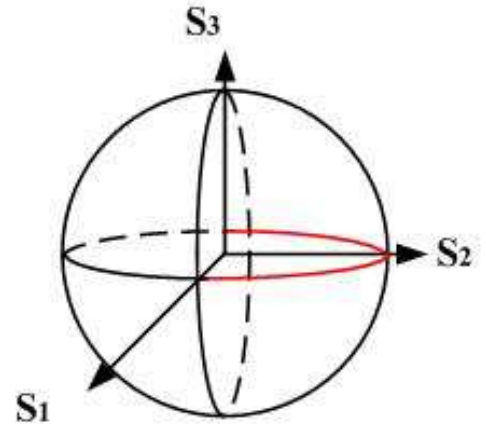

(a)

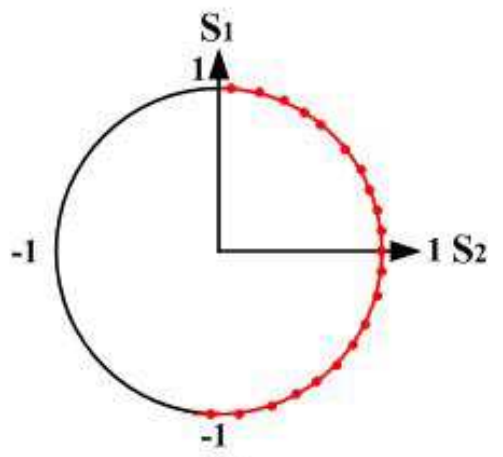

(b)

Fig. 19. Experimental measurement of the state of polarization of the output light $(\lambda=1543.47 \mathrm{~nm})$ on a Poincare sphere when varying the applied electric field from 0 to 3 $(\mathrm{kV} / \mathrm{cm})$ (Liu et al.,2009c).

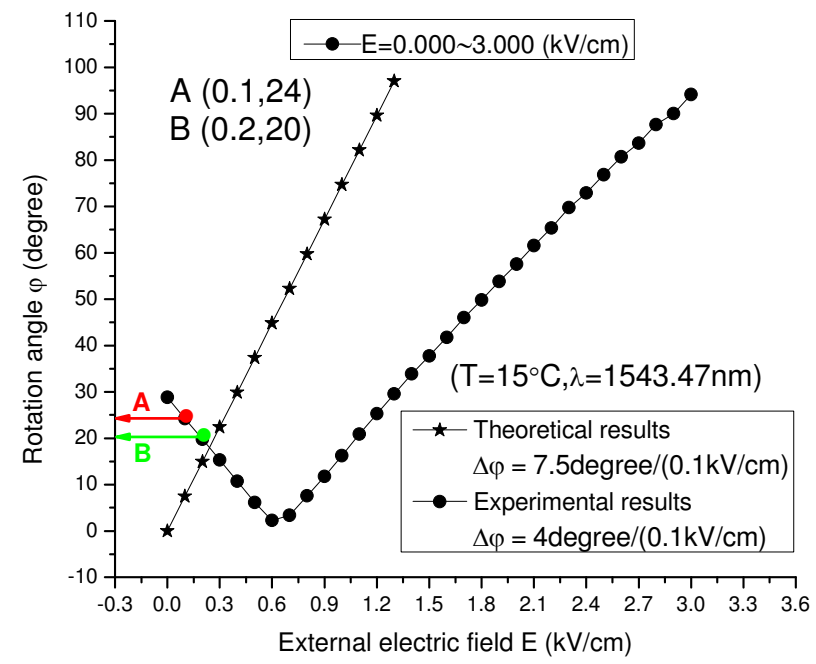

Fig. 20. Experimental measurement of the rotation angle of the output light $(\lambda=1543.47 \mathrm{~nm})$ when varying the applied electric field from 0 to $3(\mathrm{kV} / \mathrm{cm})$ (Liu et al.,2009c). 
As for the PPLN sample employed in the experiment, the operating wavelength is found out to be $\lambda=1543.47 \mathrm{~nm}$. Then, experimental measurement of the state of polarization of the output light $(\lambda=1543.47 \mathrm{~nm})$ is shown on a Poincare sphere in Fig.19 when varying the applied electric field. The experiment shows the state of polarization remains on the equatorial plane when changing the electric field which indicates the output light remains linear state of polarization. The corresponding rotation angle at each electric field is shown in Fig.20, where the electric field intensity is tuned from 0 to $3(\mathrm{kV} / \mathrm{cm})$, with the step of 0.1 $(\mathrm{kV} / \mathrm{cm})$ each time and the rotation angle linearly varies between 0-100 degrees.
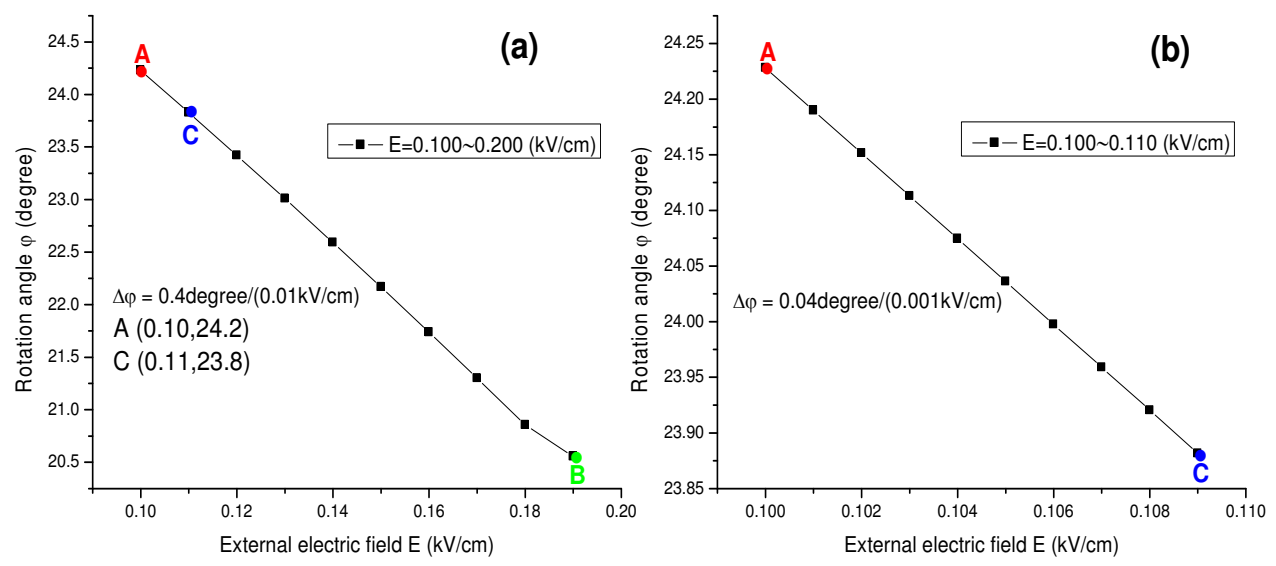

Fig. 21. Experimental measurement of the rotation angle of the output light $(\lambda=1543.47 \mathrm{~nm})$ when varying the applied electric field with smaller step. (a) The external electric field varies from 0.100 to $0.200(\mathrm{kV} / \mathrm{cm})$; (b) The external electric field varies from 0.100 to 0.110 $(\mathrm{kV} / \mathrm{cm})$ (Liu et al.,2009c).

To obtain higher precision, we reduce the electric field step to $0.01(\mathrm{kV} / \mathrm{cm})$ and 0.001 $(\mathrm{kV} / \mathrm{cm})$, respectively. Higher precision of 0.4 degree and 0.04 degree is finally achieved, limited by the accuracy of the measurement system, which are shown in Fig.21. Moreover, the operating wavelength can be tuned to a series of different wavelengths by changing the temperature.

The device operating as a precise linear polarization-state controller may find many applications where high precision control of linear polarization-state light is requested ranging from polarization analysis to monitoring of network performance, material birefringence, and measurement of polarization mode dispersion, swept-wavelength measurement, medical imaging and fiber sensor systems.

\section{Electro-optical switch}

High speed optical switch is one of the key components in optical communication. In this section, with the same experimental setup of solc-type filter, we change to with focus on the application of a precise EO switch. The first observation of an EO switch in PPLN is shown in Fig.22, where high extinction ratio with $43 \mathrm{~dB}$ is gained in the experiment. 


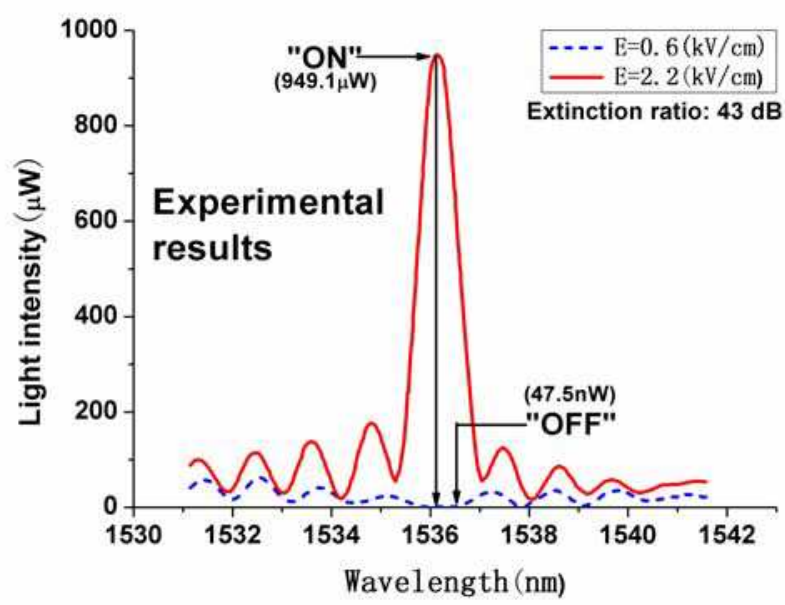

Fig. 22. The experimental transmission spectrums at electric fields of $2.2(\mathrm{kV} / \mathrm{cm})$ and 0.6 $(\mathrm{kV} / \mathrm{cm})$; The sold curve represents the switching state " $\mathrm{ON}^{\prime}$, and the dash curve represents the state "OFF".
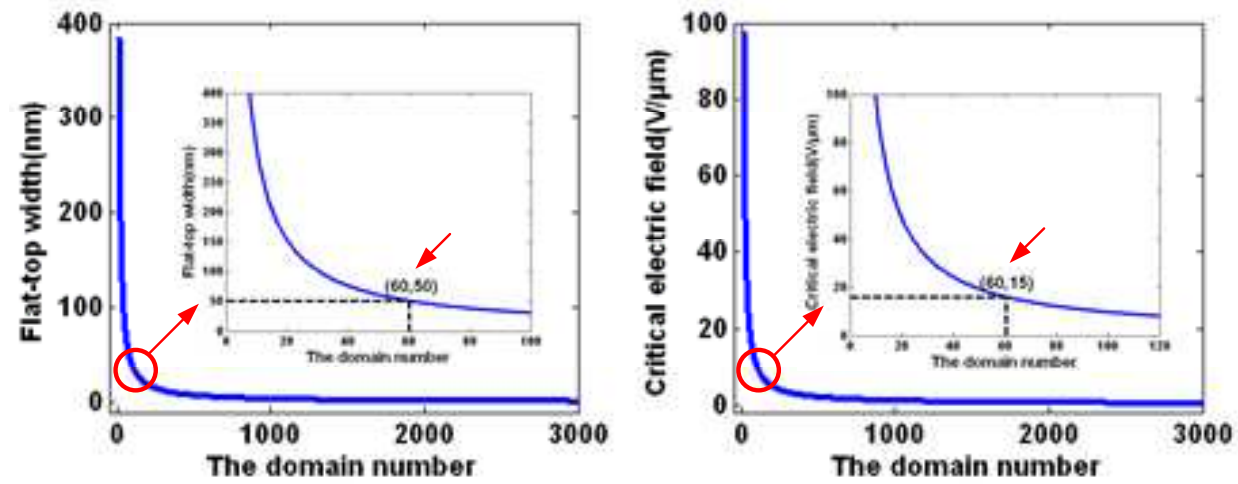

Fig. 23. (a) The flat-top width as a function of the domain number; (b) The critical electric field as a function of the domain number; The transmission spectrum at the domain number of 60 is showed in Fig.24

However, an obstacle to the application of such switch is the narrowband and wavelength limit. Inspired by the discovery of flat-top spectrum in PPLN in 2.2.3 section, we further find out a critical electric field at which the spectrum evolves into broadband. Fig. 23 shows the flat-top width and the critical electric field as a function of the domain number. Considering a given PPLN with 60 domains, the transmission spectrum at the critical electric field is shown in Fig.24, which has a broadband with $50 \mathrm{~nm}$ flat-top width. The broadband here can efficiently extend the switch's operating wavelengths. However, although sharp increase of the flat-top width can be learned from Fig.23 (a), higher width than $50 \mathrm{~nm}$ is impossible in practice, because the corresponding critical electric field will synchronously rapidly rise in Fig.23 (b), which will damage the PPLN. 


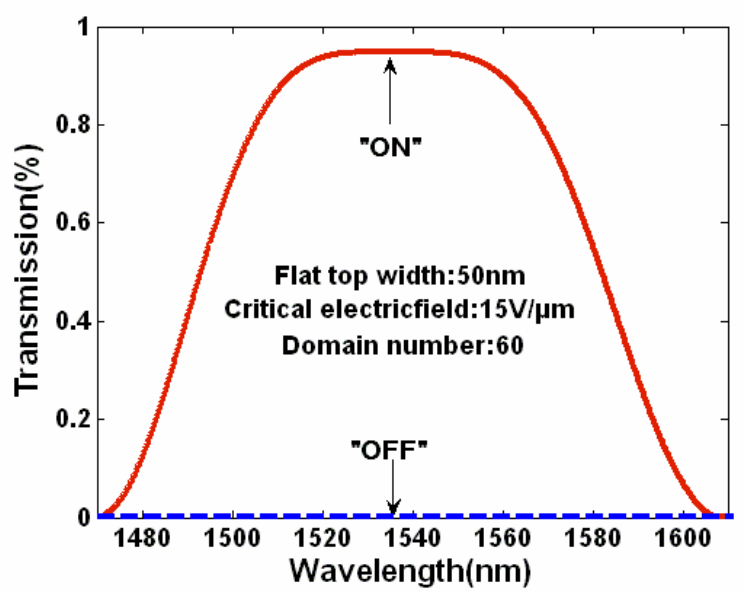

Fig. 24. The transmission spectrum at the critical electric field of $15(\mathrm{~V} / \mu \mathrm{m})$ when the domain number is 60

\section{Laser Q-switch}

A class of Q-switched lasers employs a fast intra-cavity polarization rotator for switching the laser polarization between high- and low-loss laser modes. The two loss modes can be established by using a polarization-dependent loss element such as EO PPLN crystal. In this section, we present another significant application on laser $Q$ switch laser based on EO PPLN crystal, which were proposed by Y. H. Chen et.al(Chen \& Huang,2003) and Y. Y. Lin et.al(Lin et al.,2007).

In the year of 2003, Y. H. Chen et.al successfully employed a PPLN Pockels cell as a laser Qswitch with a switching voltage as low as $\sim 100 \mathrm{~V}($ Chen \& Huang,2003). The PPLN Pockels cell consists of a quarter-wave plate and an EO PPLN crystal having the same crystal orientation as a typical PPLN wavelength converter.

Fig. 25 shows the continuous-wave (CW) performance of the Nd:YVO4 laser with and without a $100-\mathrm{V}$ voltage applied to the EO PPLN crystal. Without the applied voltage, the $\mathrm{Nd}$ :YVO4 laser had a CW threshold of 400-mW pump power, attributable to the high output coupling loss $(13 \%)$. With a 100-V voltage applied to the EO PPLN crystal, the laser's CW threshold was increased to $\sim 1.4-\mathrm{W}$ pump power as expected from the polarization rotation effect.

To show laser Q-switching operation, the EO PPLN is drove by using a 7-kHz, 100-V voltage pulser with a $300-\mathrm{ns}$ pulse width. At $1.2-\mathrm{W}$ pump power, $60-\mathrm{mW}$ average power or $0.74-\mathrm{kW}$ peak power with 8.6-mJ pulse energy in an 11.6-ns laser pulse width is measured in the experiment (Fig .26).

Despite the low switching voltage and excellent integrated QPM performance, the PPLN Pockels cell was sensitive to temperature and generated appreciable green-laser power at $532 \mathrm{~nm}$ in the Nd:YVO4 laser, both due to the QPM structure in the PPLN. It is desirable to have a "green-free" and temperature-insensitive laser Q-switch that still retains the advantages of low switching voltage and excellent integrated QPM performance. In the year 


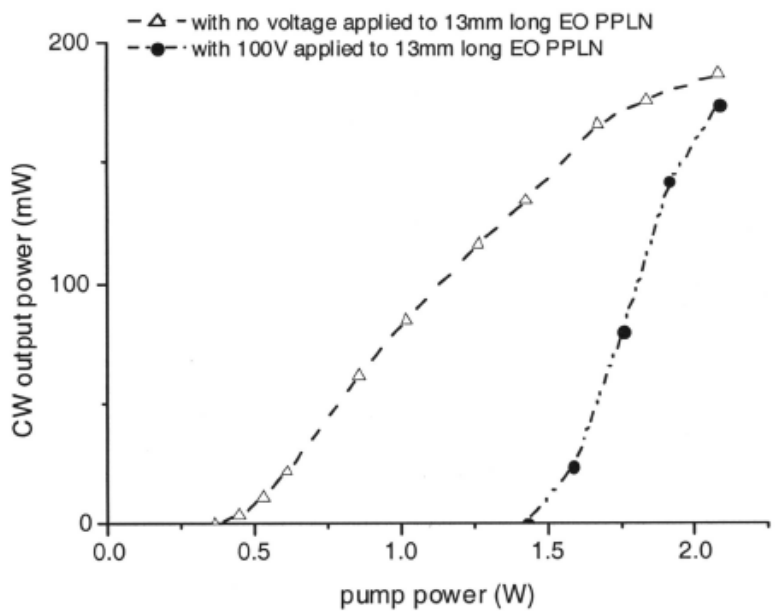

Fig. 25. CW performance of the Nd:YVO4 laser with (solid curve) and without (dashed curve) a $100-\mathrm{V}$ voltage applied to the EO PPLN. With the 100-V voltage, the laser threshold was increased from $400 \mathrm{~mW}$ to $1.4 \mathrm{~W}$ because of the polarization-rotation effect from the EO PPLN crystal(Chen \& Huang,2003).

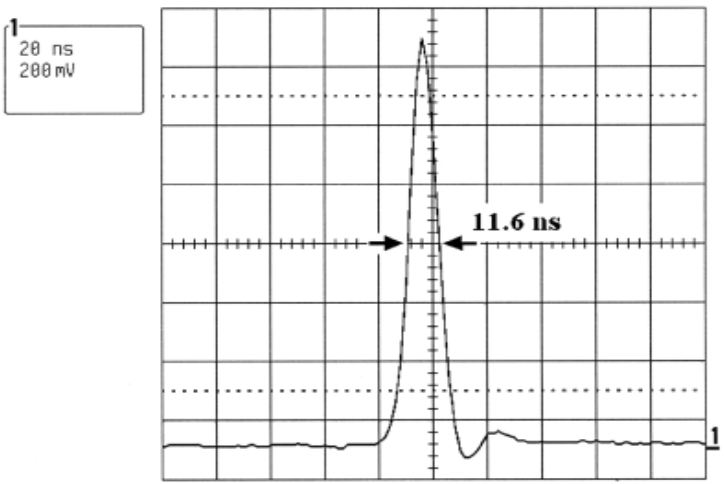

Fig. 26. Measured Q-switched pulse from the EO PPLN Q-switched Nd:YVO4 laser at 1.2-W pump power. The pulse width was $11.6 \mathrm{~ns}$, and the peak power was $\sim 0.74 \mathrm{~kW}$ (Chen \& Huang,2003).

of 2007, Y. Y. Lin et.al reported such a laser Q-switch which had overcome the previous disadvantages using a novel EO PPLN Bragg modulator, producing $7.8 \mathrm{~ns}, 201 \mu \mathrm{J}$ pulses at a $10 \mathrm{kHz}$ repetition rate when pumped by a $19.35 \mathrm{~W}$ diode laser at $808 \mathrm{~nm}$ (Y.Y.Lin et al.,2007). The schematic setups of two different kind laser Q-switches are shown in Fig.27.

Figure 28 shows the measured Q-switched pulse energy versus the diode pump power of the second kind laser Q-switch. At $19.35 \mathrm{~W}$ pump power, the Q-switched output pulse at $1064 \mathrm{~nm}$ has $201 \mu \mathrm{J}$ energy and $7.8 \mathrm{~ns}$ width, corresponding to a peak power of $26 \mathrm{~kW}$. The error bar in the plot shows that the pulse-to-pulse energy jitter was less than $5 \%$ over the range of our measurement. The inset shows the temporal profile of the Q-switched output 

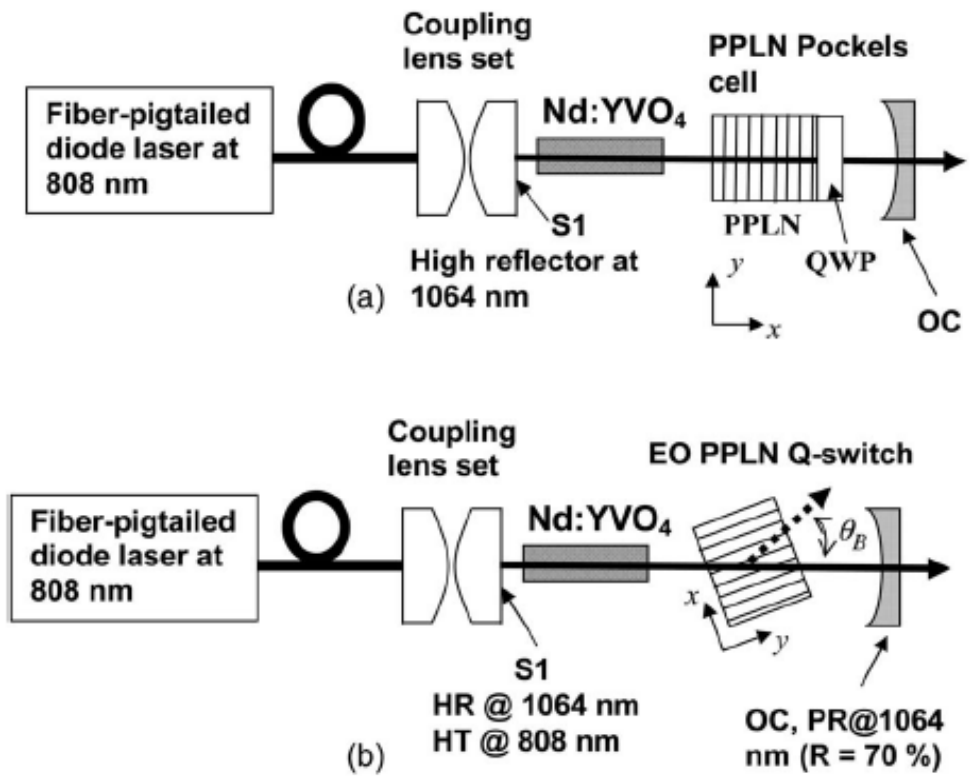

Fig. 27. Schematic of the actively Q-switched Nd:YVO4 laser using (a) a PPLN Pockels cell and (b) an EO PPLN Bragg modulator as a laser Q-switch. The laser cavity is formed by the surface $S 1$ and the output coupler (OC). In (b), the Bragg angle $\theta_{\mathrm{B}}$ is exaggerated for clarity. QWP, quarter-wave plate; HR, high reflection; HT, high transmission; PR, partial reflection(Y.Y.Lin et al,2007).

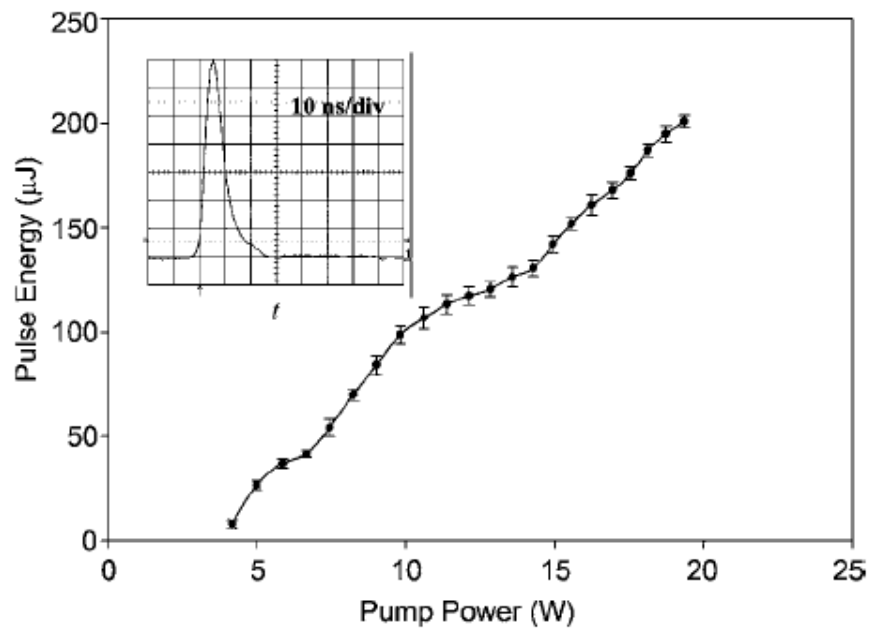

Fig. 28. Output pulse energy of the actively Q-switched Nd:YVO4 laser versus pump power. With $19.35 \mathrm{~W}$ pump power at $808 \mathrm{~nm}$, the $1064 \mathrm{~nm}$ laser pulse has an energy of $201 \sim \mathrm{J}$ and a width of $7.8 \mathrm{~ns}$, corresponding to a laser peak power of $26 \mathrm{~kW}$. The inset shows the measured temporal profile of the Q-switched laser pulse(Y.Y.Lin et al.,2007). 
pulse. In the experiment, no noticeable change was observed on the laser performance when the EO PPLN grating was heated from room temperature to $180^{\circ} \mathrm{C}$; In addition, no greenlaser power produced from the non-phase-matched second-harmonic generation in the EO PPLN grating was observed in the experiment as well. The experiment successfully confirmed the advantages of this kind Q-switch.

\section{Conclusion}

In this chapter, we have reviewed essential applications by use of polarization coupling in periodically poled lithium niobate during the past five years. Because of the outstanding properties of PPLN, we believe that PPLN will remain to be an important contemporary realm of research.

\section{References}

Chen, X.F. ; Shi, J. H.; Chen, Y. P.; Zhu, Y. M.; Xia, Y. X. \& Chen, Y. L. (2003). Electro-optic Solc-type wavelength filter in periodically poled lithium niobate, Opt. Lett. 28.

Chen, Y. H. \& Huang, Y. C. (2003). Actively Q -switched Nd:YVO4 laser using an electrooptic periodically poled lithium niobate crystal as a laser Q -switch, Opt. Lett. 28, 1460-1462.

Chen, L.J.; Shi, J.H.; Chen, X.F. \& xia, Y.X. (2006). Photovoltaic effect in a periodically poled lithium niobate Solc-type wavelength filter, Appl. Phys. Lett. 88, 121118.

$\mathrm{Gu}$, X.; Chen, X.F.; Chen, Y.P.; Zeng, X.L.; Xia, Y.X. \& Chen, Y.L. (2004). Narrowband multiple wavelengths filter in aperiodic optical superlattice, Opt. Commun.237, 5358.

Jundt, D. H. (1997). Temperature-dependent Sellmeier equation for the index of refraction, no, in congruente lithium niobate," Opt. Lett. 22, 1553-1555.

Lu, Y. Q.; Wan, Z. L.; Wang, Q.; Xi, Y. X. \& Ming, N. B. (2000). Electro-optic effect of periodically poled optical superlattice $\mathrm{LiNbO}_{3}$ and its applications, Appl. Phys. Lett. $\quad 77,3719$.

Lin, C. H.; Chen, Y. H.; Lin, S. W.; Chang, C. L.; Huang, Y. C. \& Chang, J. Y. (2007). Electrooptic narrowband multi-wavelength filter in aperiodically poled lithium niobate, Opt. Exp. 15, 9859-986.

Lee, Y. L.; Yu, N. E.; Kee, C. -.; Ko, D. -.; Lee, J.; Yu, B. -.; Shin, W.; Eom, T. J. \& Noh, Y. -. (2007). Wavelength filtering characteristics of Solc filter based on Ti:PPLN channel waveguide, Opt. Lett. 32, 2813-2815.

Lee, Y. L.; Yu, N.E.; Kee, C.-S.; Ko, D.-K.; Noh, Y.-C.; Yu, B.-A.; Shin, W.; Eom, T.-J.; Oh, K. \& Lee, J. (2008). All-optical wavelength tuning in Solc filter based on Ti:PPLN waveguide, Electron. Lett. 44, 30-32.

Lin, Y.Y.; Lin, S.T.; Chang, G.W.; Chiang, A.C.; Huang, Y.C. \& Chen, Y.H. (2007). Electrooptic periodically poled lithium niobate Bragg modulator as a laser Q-switch, Opt. Lett. 32, 545-547.

Liu, K.; Shi, J.H.; Zhou, Z.E. \& Chen, X.F. (2009a). Electro-optic Solc-type flat-top bandpass filter based on periodically poled lithium niobate, Opt. Commun.282, 1207-1211.

Liu, K.; Shi, J.H. \& Chen, X.F. (2009b). Electro-optical flat-top bandpass Solc-type filter in periodically poled lithium niobate. Opt. Lett. 34, 7. 
Liu, K.; Shi, J.H. \& Chen, X.F. (2009c). Linear polarization-state generator with high precision in periodically poled lithium niobate, Appl. Phys. Lett., 94, 101106.

Magel, G. A.; Fejer, M. M. \& Byer, R. L. (1990). Quasi-phase-matched second-harmonic generation of blue light in periodically poled $\mathrm{LiNbO}_{3}$, Appl. Phys. Lett. 56, 108-110.

Shi, J. H.; Wang, J. H.; Chen, L. J.; Chen, X. F. \& Xia, Y. X. (2006). Tunable Šolc-type filter in periodically poled LiNbO3 by UV-light illumination, Opt. Exp. 14, 6279.

Wang, J. H.; Shi, J. H.; Zhou, Z. E. \& Chen, X. F. (2007). Tunable multi-wavelength filter in periodically poled $\mathrm{LiNbO} 3$ by a local-temperature-control technique, Opt. Exp. 15,1561 .

Xu, C. Q.; Okayama, H. \& Kawahara, M. (1993). 1. $5 \mu \mathrm{m}$ band efficient broadband wavelength conversion by difference frequency generation in a periodically domain-inverted $\mathrm{LiNbO}_{3}$ channel waveguide, Appl. Phys. Lett. 63, 3559-3561.

Yariv, A. \& Yeh, P. (1984). Optical Waves in Crystal: Propagation and Control of Laser Radiation (Wiley, NewYork).

Zheng, J. J.; Lu, Y. Q.; Luo, G.P. ; Ma, J.; Lu, Y. L.; Ming, N. B.; He, J. L. \& Xu, Z. Y. (1998). Visible dual-wavelength light generation in optical superlattice through Er: $\mathrm{LiNbO}_{3}$ unconversion and quasi-phase-matched frequency doubling, Appl. Phys. Lett.72, 1808-1810.

Zhu, Y. M.; Chen, X. F.; Shi, J. H.; Chen, Y. P.; Xia, Y. X. \& Chen, Y. L. (2003). Wide-range tunable wavelength filter in periodically poled lithium niobate, Opt. Comm., 228,139-143. 


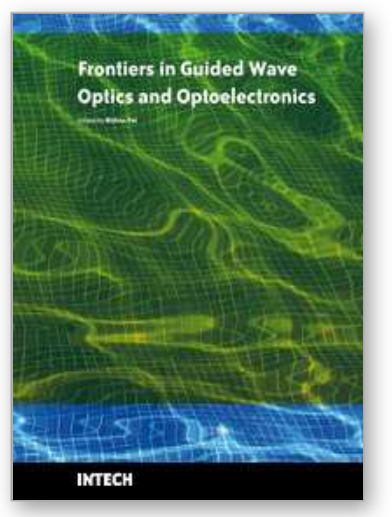

\author{
Frontiers in Guided Wave Optics and Optoelectronics \\ Edited by Bishnu Pal
}

ISBN 978-953-7619-82-4

Hard cover, 674 pages

Publisher InTech

Published online 01, February, 2010

Published in print edition February, 2010

As the editor, I feel extremely happy to present to the readers such a rich collection of chapters authored/coauthored by a large number of experts from around the world covering the broad field of guided wave optics and optoelectronics. Most of the chapters are state-of-the-art on respective topics or areas that are emerging. Several authors narrated technological challenges in a lucid manner, which was possible because of individual expertise of the authors in their own subject specialties. I have no doubt that this book will be useful to graduate students, teachers, researchers, and practicing engineers and technologists and that they would love to have it on their book shelves for ready reference at any time.

\title{
How to reference
}

In order to correctly reference this scholarly work, feel free to copy and paste the following:

Xianfeng Chen, Kun Liu, and Jianhong Shi (2010). Polarization Coupling of Light and Optoelectronics Devices Based on Periodically Poled Lithium Niobate, Frontiers in Guided Wave Optics and Optoelectronics, Bishnu Pal (Ed.), ISBN: 978-953-7619-82-4, InTech, Available from: http://www.intechopen.com/books/frontiers-in-guidedwave-optics-and-optoelectronics/polarization-coupling-of-light-and-optoelectronics-devices-based-onperiodically-poled-lithium-nioba

\section{INTECH}

open science | open minds

\section{InTech Europe}

University Campus STeP Ri

Slavka Krautzeka 83/A

51000 Rijeka, Croatia

Phone: +385 (51) 770447

Fax: +385 (51) 686166

www.intechopen.com

\section{InTech China}

Unit 405, Office Block, Hotel Equatorial Shanghai

No.65, Yan An Road (West), Shanghai, 200040, China

中国上海市延安西路65号上海国际贵都大饭店办公楼 405 单元

Phone: +86-21-62489820

Fax: +86-21-62489821 
(C) 2010 The Author(s). Licensee IntechOpen. This chapter is distributed under the terms of the Creative Commons Attribution-NonCommercialShareAlike-3.0 License, which permits use, distribution and reproduction for non-commercial purposes, provided the original is properly cited and derivative works building on this content are distributed under the same license. 